\title{
Beneficial effects of early administration of recombinant human B-type natriuretic peptide in ST-elevation myocardial infarction patients receiving percutaneous coronary intervention treatment
}

Lijun Wang ${ }^{1}, \mathrm{MD}$, Lianna Xie ${ }^{1}, \mathrm{MD}$, Xianjing We ${ }^{1}, \mathrm{MD}$, Zezhou Xie ${ }^{1}, \mathrm{MD}$

\begin{abstract}
INTRODUCTION We aimed to evaluate the clinical performance of early administration of recombinant human B-type natriuretic peptide (rhBNP) to ST-elevation myocardial infarction (STEMI) patients receiving percutaneous coronary intervention $(\mathrm{PCl})$ treatment.

METHODS In total, 185 patients diagnosed with STEMI were enrolled and randomised into either the placebo-treated $(n=88)$ or rhBNP-treated $(n=97)$ group. Patients were given either saline or rhBNP ten minutes before $\mathrm{PCl}$ and monitored with various cardiac parameters, including accelerated idioventricular rhythm, frequent ventricular premature beat (FVPB), ventricular tachycardia, systolic blood pressure, thrombolysis in myocardial infarction (TIMI) 3 gradation, corrected TIMI frame count (CTFC) and myocardial blush grade (MBG) 3 classification.

RESULTS Our results revealed no difference in accelerated idioventricular rhythm between the two groups. However, FVPB and ventricular tachycardia were significantly decreased in rhBNP-treated patients compared to placebo-treated patients $(p<0.05)$. Moreover, the occurrence ratio of reperfusion-associated low blood pressure in rhBNP-treated patients was lower than in placebo-treated patients $(p=0.03)$, while no difference was observed in infarction-related arteries TIMI 3 blood flow between the two groups $(p=0.23)$. Importantly, measurement of post-reperfusion blood flow velocity via CTFC suggested that rhBNP treatment could significantly increase blood circulation $(p=0.003)$. After stent implantation, the acquisition rate of MBG 3 was higher in rhBNP-treated patients compared to placebo-treated patients $(p=0.071)$, although the difference was not significant.

CONCLUSION We concluded that early administration of rhBNP can ameliorate the severity of reperfusion injury for STEMI patients receiving PCI treatment.
\end{abstract}

Keywords: anterior wall myocardial infarction, coronary blood flow, reperfusion injury, rhBNP

\section{INTRODUCTION}

Direct percutaneous coronary intervention $(\mathrm{PCl})$ is the most timely and effective reperfusion method for treating acute ST-elevation myocardial infarction (STEMI), as it efficiently increases blood flow in infarction-related arteries (IRA). However, $\mathrm{PCl}$ is unable to prevent reperfusion injury. ${ }^{(1,2)}$ Reperfusion arrhythmia (RA), one of the earliest manifestations of reperfusion injury, may result in fatal consequences if it is not treated in a timely fashion. ${ }^{(3)}$ Reperfusionassociated low blood pressure and slow blood flow can impair myocardial perfusion, which further aggravates myocardial injury and affects short- and long-term prognoses. ${ }^{(4)}$ Thus, it has become pivotal to explore novel medical treatments to reduce myocardial reperfusion injury.

Recombinant human B-type natriuretic peptide (rhBNP) is a synthetic peptide with similar biological activity to endogenous B-type natriuretic peptide (BNP), which was approved by the United States Food and Drug Administration for the treatment of acute decompensated heart failure in 2001. ${ }^{(5)}$ However, a recent clinical trial could not confirm the benefit of rhBNP in patients with acute decompensated heart failure, although the study excluded renal toxicity and the mortality associated with its use. ${ }^{(6)}$ Previously, rhBNP had been proven to play multiple functions, such as facilitating natriuresis and diuresis, inhibiting the renin-angiotensin-aldosterone system, increasing cardiac output, decreasing pulmonary capillary wedge pressure and improving diastolic function. ${ }^{(7-10)}$ In this study, we aimed to explore the potential effects of early administration of rhBNP in STEMI patients receiving direct $\mathrm{PCl}$ reperfusion and to evaluate its therapeutic value in ameliorating myocardial ischaemia-reperfusion injury.

\section{METHODS}

Patients who were diagnosed with STEMI and were receiving emergent PCl treatment in Dalian University, Liaoning, China, from January 2013 to December 2015 were enrolled in this study. The inclusion criteria were: (a) age 45-75 years; (b) met the diagnostic criteria for acute anterior wall STEMI; (c) diagnosed within 12 hours of the onset of symptoms; (d) approval of the patient or family members for direct PCI treatment; and (e) fully written informed consent to receive rhBNP. Patients were excluded from this study if any of the following criteria was present: (a) cardiogenic shock or systolic blood pressure (SBP) $<100 \mathrm{mmHg}$; (b) IRA blood flow thrombolysis in myocardial 
infarction (TIMI) Grade 2-3 according to coronary angiography; (c) severe deficiency in liver and kidney function; or (d) allergy to rhBNP. All procedures involving human participants were approved by the Ethics Committee of Dalian University.

Patients who met the inclusion criteria were randomly assigned to either the placebo-treated or rhBNP-treated group using covariate adaptive randomisation (http://www.graphpad. com/quickcalcs/index.cfm). Patients in the placebo-treated group received regular medication for STEMI, saline administration and emergent $\mathrm{PCl}$, while patients in the rhBNP-treated group received regular medication for STEMI, rhBNP treatment and emergent $\mathrm{PCl}$. The rhBNP administration protocol was as follows: ten minutes before $\mathrm{PCl}$, the patient was given rhBNP $(1.5 \mu \mathrm{g} / \mathrm{kg}$ body weight) via intravenous injection for three minutes; subsequently, $0.01 \mu \mathrm{g} / \mathrm{min} / \mathrm{kg}$ was continuously administered until a total of $0.5 \mathrm{mg}$ was reached.

We collected the patients' demographics and clinical information, including age, gender, presence of hypertension and diabetes mellitus, history of smoking and myocardial infarction, preoperative Killip class, intraoperative application of suction catheter, reperfusion times and door-to-balloon time. To define the coronary artery lesions, we also recorded cardiac parameters such as the number of coronary artery lesions, IRA position and TIMI blood flow before surgery.

We used electrocardiography to monitor post RA, frequent ventricular premature beat (FVPB), accelerated idioventricular rhythm, ventricular tachycardia, ventricular fibrillation and severe slow arrhythmia from the beginning of reperfusion to the end of $\mathrm{PCl}$. To determine post-reperfusion blood pressure, we recorded the lowest stable invasive blood pressure indicated on the recording instrument within 15 minutes after reperfusion.

Coronary angiography was used to assess post-reperfusion TIMI blood flow. The criteria were as follows - Grade 0 (no perfusion): vessel occlusion with no forward flow on distal end; Grade 1 (with permeation but no perfusion): the contrast agent passed the occluded position but failed to fill the distal vessel; Grade 2 (partial perfusion): the contrast agent fully filled the distal coronary artery at a lower speed of filling and eliminating as compared to that in a normal coronary artery; and Grade 3 (full perfusion): the contrast agent fully and rapidly filled the distal vessel, and was eliminated quickly.

For corrected TIMI frame count (CTFC) analysis, we used the concrete method, as previously described. ${ }^{(11)}$ In brief, nitroglycerin $200 \mu \mathrm{g}$ was injected into the coronary artery and CTFC was then measured by coronary angiography when the IRA forward flow reached TIMI 3. The frame number index was measured when the contrast agent passed the unit vessel, and the frame number was recorded from the time the contrast agent filled the artery to the time it reached the mark on the distal vessel. ${ }^{(11)}$

To examine the graded changes of myocardial perfusion, we applied the myocardial blush grades (MBG) criteria proposed by the Zwolle Myocardial Infarction Study Group in 1998. ${ }^{(12)}$ The grades were defined as follows - Grade 0: no penetration of the contrast agent into the myocardium or no contrast agent density, indicating no perfusion in the myocardium; Grade 1: minimal penetration of the contrast agent into the myocardium or minimal contrast agent density, indicating a little perfusion in the myocardium; Grade 2: moderate penetration of the contrast agent into the myocardium or moderate contrast agent density less than that on the same side or opposite side of the IRA, indicating partial reperfusion in the myocardium; and Grade 3: normal penetration of the contrast agent into the myocardium or normal contrast agent density equal to that on the same side or opposite side of the IRA, indicating complete reperfusion in the myocardium.

All data was analysed using PASW Statistics version 18.0 (SPSS Inc, Chicago, IL, USA). Categorical variables were analysed using chi-square or Fisher's exact test, and these were shown as percentage. Continuous variables were analysed using Student's $t$-test if normal distribution of data was confirmed by the Kolmogorov-Smirnov test; otherwise, Mann-Whitney $U$ test was used. Data was presented as mean \pm standard error of mean, unless otherwise specified. A p-value $<0.05$ was considered statistically significant.

\section{RESULTS}

A total of 185 patients were recruited for the study. They were randomised into the placebo-treated group $(n=88)$ and the rhBNP-treated group $(n=97)$. The demographics and clinical information of the patients are summarised in Table I. No significant demographic and clinical differences were observed between the two groups, suggesting that our analysis setting was unbiased. Characteristics of the coronary artery lesions in the two patient groups are summarised in Table II. We observed no significant differences between the placebo-treated and rhBNPtreated groups in terms of cardiac parameters, suggesting a lack of sampling bias in our study.

Our results showed that early administration of rhBNP reduced the occurrence of post RA. Compared to the placebotreated group, we observed no significant difference in accelerated idioventricular rhythm in the rhBNP-treated group (Fig. 1a). The patients who received rhBNP showed significantly reduced FVPB and ventricular tachycardia ratio $(p<0.05$; Figs. $1 \mathrm{~b} \& \mathrm{c}$ ). Additionally, in the placebo-treated group, one patient had ventricular fibrillation and another had third-degree atrioventricular blocking. In contrast, malignant ventricular arrhythmia or severe slow arrhythmia did not occur in the rhBNPtreated group.

We also found that rhBNP treatment reduced the incidence of post-reperfusion-associated low blood pressure. Using SBP as an indicator, 51 (57.95\%) patients in the placebo-treated group and $76(78.35 \%)$ patients in the rhBNP-treated group had SBP $\geq 90 \mathrm{mmHg}$ ( $p=0.06$; Fig. 2a). In the rhBNP-treated group, there were significantly fewer patients whose SBP was 60-90 mmHg compared to the placebo-treated group $(21.86 \%$ vs. $39.47 \%$, $\mathrm{p}=0.03$; Fig. 2b). There was one patient in the placebo-treated group with SBP $<60 \mathrm{mmHg}$. These observations suggested that early rhBNP treatment could decrease post-reperfusion-associated hypotension in STEMI patients.

Coronary blood flow velocity and myocardial microcirculation perfusion were found to be increased in the rhBNP-treatment 
Table I. Demographics and clinical information of the two patient groups.

\begin{tabular}{|c|c|c|c|}
\hline \multirow[t]{2}{*}{ Variable } & \multicolumn{2}{|c|}{ No. (\%) } & \multirow[t]{2}{*}{ p-value } \\
\hline & Placebo-treated $(\mathbf{n}=\mathbf{8 8})$ & rhBNP-treated $(n=97)$ & \\
\hline Male gender & $64(72.73)$ & $73(75.26)$ & 0.58 \\
\hline $\operatorname{Age}^{*}(y r)$ & $63.13 \pm 9.47$ & $61.95 \pm 8.30$ & 0.37 \\
\hline Hypertension & $49(55.68)$ & $59(60.82)$ & 0.51 \\
\hline Diabetes mellitus & 29 (32.95) & $27(27.84)$ & 0.51 \\
\hline History of smoking & $46(52.27)$ & $56(57.73)$ & 0.33 \\
\hline History of myocardial infarction & $8(9.09)$ & $7(7.22)$ & 0.54 \\
\hline Killip Class II-III & $25(28.41)$ & $27(27.84)$ & 0.52 \\
\hline Intraoperative thrombus suction & $53(60.23)$ & $54(55.67)$ & 0.44 \\
\hline Time of reperfusion* (hr) & $5.67 \pm 2.18$ & $5.89 \pm 2.52$ & 0.60 \\
\hline
\end{tabular}

${ }^{*}$ Data presented as mean \pm standard error of mean. rhBNP: recombinant human B-type natriuretic peptide

Table II. Characteristics of coronary artery lesions in the two patient groups.

\begin{tabular}{|c|c|c|c|}
\hline \multirow[t]{2}{*}{ Characteristic } & \multicolumn{2}{|c|}{ No. (\%) } & \multirow[t]{2}{*}{ p-value } \\
\hline & Placebo-treated $(n=88)$ & rhBNP-treated $(n=97)$ & \\
\hline \multicolumn{4}{|l|}{ No. of lesions } \\
\hline 3 branches & 19 (21.59) & $24(24.74)$ & 0.44 \\
\hline 2 branches & $41(46.59)$ & $50(51.55)$ & 0.50 \\
\hline \multicolumn{4}{|l|}{ IRA location } \\
\hline LADm & $42(47.73)$ & $41(42.27)$ & 0.49 \\
\hline \multicolumn{4}{|l|}{ TIMI blood flow } \\
\hline Grade 0 & $80(90.91)$ & $90(92.78)$ & 0.55 \\
\hline Grade 1 & $10(11.36)$ & $10(10.31)$ & 0.52 \\
\hline
\end{tabular}

IRA: infarction-related arteries; LADm: left anterior descending middle; LADp: left anterior descending proximal; rhBNP: recombinant human B-type natriuretic peptide; TIMI: thrombolysis in myocardial infarction

group. The proportion of patients with TIMI 3 following PCI was higher in the rhBNP-treated group as compared to the placebotreated group $(96.86 \%$ vs. $89.47 \%, p=0.23$; Fig. 3a). Coronary blood flow velocity, as indicated by cTFC analysis, was also significantly higher in the rhBNP-treated group $(p=0.003$; Fig. 3b). Furthermore, although not significant, the acquisition ratio of MBG 3 was higher in the rhBNP-treated group than in the placebo-treated group $(75.00 \%$ vs. $55.26 \%, p=0.071$; Fig. 3c), suggesting that rhBNP treatment could improve not only haemodynamic status, but also myocardial microcirculation perfusion, in STEMI patients who received $\mathrm{PCI}$ treatment.

\section{DISCUSSION}

It is well known that the highest priority of STEMI treatment is the restoration of blood flow as quickly as possible. However, blood reperfusion can be detrimental, as it can induce severe tissue damage in the ischaemic myocardium, which is also known as ischaemia-reperfusion injury. One of the major complications of reperfusion injury that occurs in acute myocardial infarction patients is RA, which is induced by autolysis, thrombolysis in coronary arteries, $\mathrm{PCl}$ treatment or other clinical methods. The incidence of RA is usually higher in PCl-treated patients when the arteries are opened, but some cases of RA may also result in fatal consequences if not treated in a timely manner. Reperfusion injuries usually correlate with poor myocardial microcirculation, which can lead to myocardium dysfunction and cardiac electrophysiological disorder, thus affecting the patient's cardiac function and prognosis. As early as 1996, the effects of reperfusion injury were verified in animal models. ${ }^{(13)}$

The major culprit in myocardial ischaemia-reperfusion injury is the production of free radicals and their lipid peroxidation effects, which ultimately lead to harmful electrophysiological changes. ${ }^{(14,15)}$ With the influx of a large amount of oxygen during myocardial ischaemic reperfusion, the massive oxygen free radicals produced can attack biological membranes, resulting in lipid peroxidation, unstable membrane potentials, inhomogeneous depolarisations and repolarisations, as well as severe arrhythmia repolarisation. ${ }^{(16,17)}$ Moreover, myocardial ischaemia can inhibit adenosine triphosphate (ATP) synthesis and decrease the sodiumexcreting and potassium-sparing ability in the cardiomyocytes. When blood flow restores, large amounts of sodium and calcium enter the cells and lead to calcium overload, which potentiates myocardial cell injury and ultimately leads to RA.

BNP is an important heart hormone that consists of 32 amino acids. It activates the second messenger cyclic guanosine monophosphate (cGMP) by binding to its cognate natriuretic peptide receptors, thus initiating a series of physiological effects. These include a decrease in systemic vascular resistance and central venous pressure, and an increase in natriuresis. It has been demonstrated that BNP secretion is a protective compensatory 

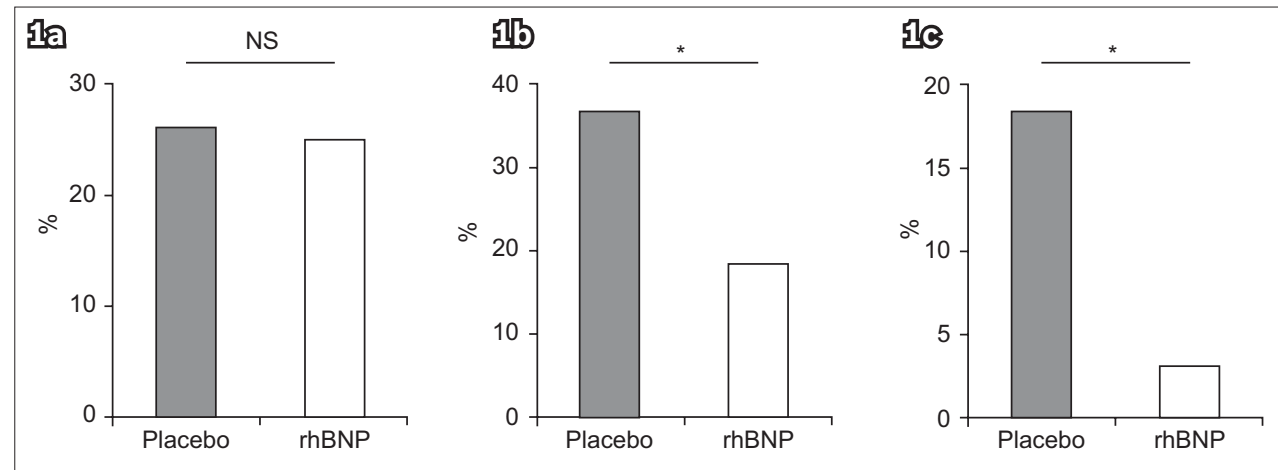

Fig. 1 Bar graphs show the comparison of (a) accelerated idioventricular rhythm; (b) frequent ventricular premature beat; and (c) ventricular tachycardia between the placebo-treated and rhBNP-treated groups. ${ }^{*} \mathrm{p}<0.05$; NS: not significant; rhBNP: recombinant human B-type natriuretic peptide

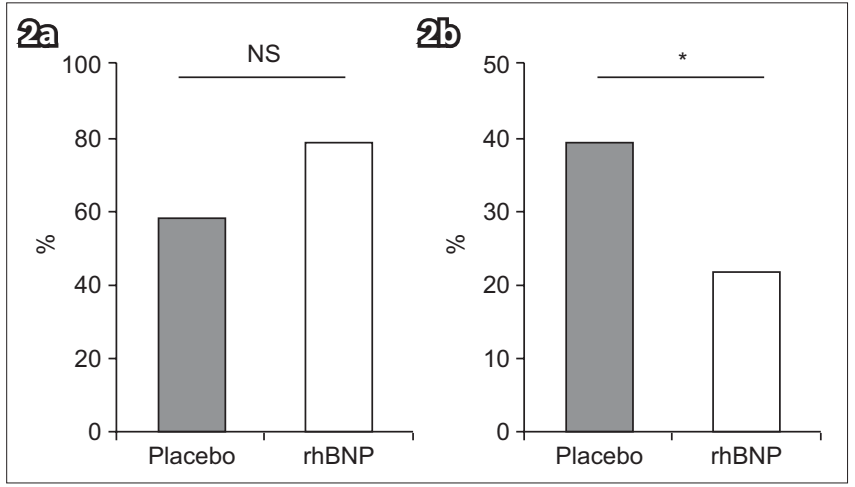

Fig. 2 Bar graphs show the comparison of (a) SBP $\geq 90 \mathrm{mmHg}$; (b) $60 \leq$ $\mathrm{SBP}<90 \mathrm{mmHg}$ between the placebo-treated and rhBNP-treated groups. ${ }^{*} p<0.05$ is considered significant. NS: not significant; rhBNP: recombinant human B-type natriuretic peptide; SBP: systolic blood pressure

response of cardiomyocytes against a variety of pathological lesions. ${ }^{(18)}$ rhBNP is structurally and biochemically identical to endogenous BNP. ${ }^{(17)}$ The interaction between rhBNP and its cognate receptor can activate guanylyl cyclase, increasing its concentration in cells. High levels of guanylyl cyclase can reduce the intracellular calcium concentration and increase antioxidant response ability, both of which help to ameliorate the oxidative damage of cardiomyocytes induced by free radicals. Furthermore, rhBNP can also open up ATP-sensitive potassium channels through the CGMP-dependent protein kinase pathway, increasing the influx of potassium into the mitochondria and reducing the difference in transmembrane potential and the influx of calcium. Thus, rhBNP can effectively reduce calcium overload in myocardial mitochondria during ischaemia reperfusion, thereby enhancing myocardial anti-ischaemic and hypoxia capacity. ${ }^{(19)}$

Using the canine myocardial reperfusion injury models, Takata et al demonstrated that intravenous injections of atrial natriuretic peptide (ANF), as compared to the administration of glucose in controls, can markedly increase plasma cGMP levels and significantly decrease the incidence of various pathological types of ventricular arrhythmia. ${ }^{(13)}$ In addition, as a reninangiotensin-aldosterone system blocker, BNP can antagonise the sympathetic nervous system and the cardiac toxic effect induced by excessive neuroendocrine activation. Indeed, it has been reported that intravenous injections of ANF can reduce cardiac sympathetic nerve activity and ischaemia-reperfusion injury in patients with acute myocardial infarction. ${ }^{(20)}$ Similarly, our data suggests that compared to a placebo, rhBNP treatment can significantly reduce the incidence of FVPB and ventricular tachycardia after reperfusion in STEMI patients who received $\mathrm{PCl}$ treatment $(\mathrm{p}<0.05)$, suggesting that rhBNP can decrease the risk of RA.

Reperfusion hypotension often occurs after acute myocardial infarction reperfusion. Serious reperfusion hypotension may cause haemodynamic disorders that affect the perfusion of vital organs and increase surgical risk. The pathogenic mechanism of hypotension may be associated with myocardial stunning after opening of the left anterior descending artery. ${ }^{(21)}$ In the present study, we also reported that administration of rhBNP can significantly decrease the incidence of post-reperfusion-associated hypotension compared to a placebo $(p<0.05)$, which may be due to the fact that rhBNP is known to improve ischaemic myocardial microcirculation perfusion. ${ }^{(22)}$

Michaels et al have reported that administration of rhBNP can rapidly improve haemodynamic parameters, including increasing coronary blood supply, dilating the coronary artery diameter and decreasing circulation resistance to provide sufficient blood perfusion to the myocardial infarction area. ${ }^{(22)}$ In line with this, our study, which used cTFC to estimate coronary blood flow velocity after rhBNP administration, showed that the coronary blood flow velocity in the rhBNP-treated group was significantly higher than that in the placebo-treated group $(27.24 \% \pm 0.61 \%$ vs. $24.63 \% \pm 0.57 \% ; p=0.003)$. This demonstrates that rhBNP could efficiently increase efficient myocardial reperfusion in STEMI patients receiving PCI treatment. We also used the MBG method to determine myocardial microcirculation perfusion efficiency and found that the myocardial perfusion level of the rhBNP-treated group was higher than that of the placebo-treated group, although it was not significantly different. Taken together, our findings demonstrate that early application of rhBNP not only markedly improves the coronary flow velocity, but also increases myocardial microcirculation perfusion in STEMI patients receiving $\mathrm{PCl}$ treatment.

Admittedly, our study focused only on the effects of early application of rhBNP on reperfusion response but did not utilise more specific measurements, such as magnetic resonance imaging assessment to evaluate myocardium at risk and the final size of 


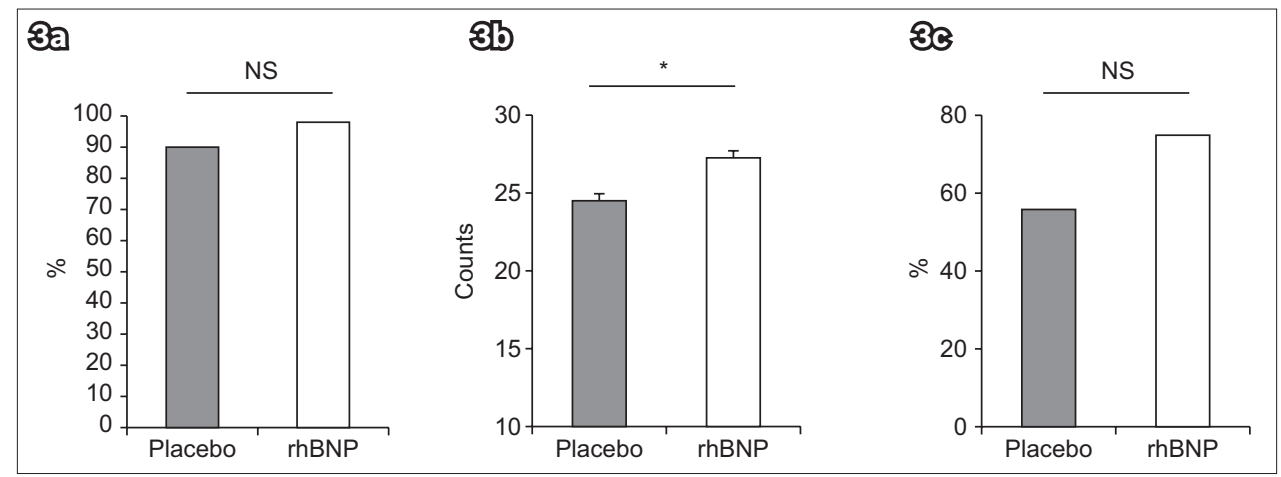

Fig. 3 Bar graphs show the comparison of (a) TIMI grade 3; (b) corrected TIMI frame count; and (c) myocardial blush grade 3 between the placebo-treated and rhBNP-treated groups. ${ }^{*} \mathrm{p}<0.05$; NS: not significant; rhBNP: recombinant human B-type natriuretic peptide; TIMI: thrombolysis in myocardial infarction

myocardial infarcts, or 18F-sodium fluoride positron emission tomography to evaluate myocardium damage and necrosis, due to technical issues. Moreover, the number of the patients involved in this study was relatively small and the study was not carried out in an absolutely double-blind fashion. Therefore, it is necessary to expand this area of investigation using a larger sample size in order to verify the beneficial effects of early administration of rhBNP in STEMI patients receiving PCI treatment.

In conclusion, this study showed that early application of rhBNP can reduce the occurrence of reperfusion ventricular arrhythmias and reperfusion-associated hypotension, as well as concurrently improve coronary blood flow and myocardial microcirculation perfusion. Therefore, rhBNP confers multiple beneficial effects on STEMI patients receiving $\mathrm{PCI}$ treatment.

\section{ACKNOWLEDGEMENTS}

We thank the cardiologists and colleagues in our department for their kind support and input. The study was supported by the 2013 Medical and Health Science Research Program of Dalian (2007-530) and registered with the Chinese Clinical Trials registry (ChiCTR-TRC-08000072).

\section{REFERENCES}

1. Estévez-Loureiro R, López-Sainz A, Pérez de Prado A, et al. Timely reperfusion for ST-segment elevation myocardial infarction: effect of direct transfer to primary angioplastyon time delays and clinical outcomes. World J Cardiol 2014; 6:424-33.

2. PiérardLA. ST elevation after myocardial infarction: what does it mean? Heart 2007; 93:1329-30.

3. Eltzschig HK, Collard CD. Vascular ischaemia and reperfusion injury. Br Med Bull2004; 70:71-86.

4. Eltzschig HK, Eckle T. Ischemia and reperfusion--from mechanism to translation. Nat Med 2011; 17:1391-401.

5. Keating GM, Goa KL. Nesiritide: a review of its use in acute decompensated heart failure. Drugs 2003; 63:47-70.

6. O'Connor CM, Starling RC, Hernandez AF, et al. Effect of nesiritide in patients with acute decompensated heart failure. N Engl J Med 2011; 365:32-43.

7. Young JB, Cheng M, Mills RM. Hemodynamics, diuretics, and nesiritide: a retrospective VMAC analysis. Clin Cardiol 2009; 32:530-6.

8. Elkayam U, Akhter MW, Singh H, Khan S, Usman A. Comparison of effects on left ventricular filling pressure of intravenous nesiritide and high-dose nitroglycerin in patients with decompensated heart failure. Am J Cardiol 2004; 93:237-40.

9. Publication Committee for the VMAC Investigators (Vasodilatation in the Management of Acute CHF). Intravenous nesiritide vs nitroglycerin for treatment of decompensated congestive heart failure: a randomized controlled trial. JAMA 2002; 287:1531-40.

10. Colucci WS, Elkayam U, Horton DP, et al. Intravenous nesiritide, a natriuretic peptide, in the treatment of decompensated congestive heart failure. Nesiritide Study Group. N Engl J Med 2000; 343:246-53.

11. Bellandi F, Leoncini M, Maioli M, et al. Markers of myocardial reperfusion as predictors of left ventricular function recovery in acute myocardial infarction treated with primary angioplasty. Clin Cardiol 2004; 27:683-8.

12. van't Hof AW, Liem A, Suryapranata H, et al. Angiographic assessment of myocardial reperfusion in patients treated with primary angioplasty for acute myocardial infarction: myocardial blush grade. Zwolle Myocardial Infarction Study Group. Circulation 1998; 97:2302-6.

13. Takata $Y$, Hirayama $Y$, Kiyomi S, et al. The beneficial effects of atrial natriuretic peptide on arrhythmias and myocardial high-energy phosphates after reperfusion. Cardiovasc Res 1996; 32:286-93.

14. Zhao W, Fan GC, Zhang ZG, et al. Protection of peroxiredoxin II on oxidative stress-induced cardiomyocyte death and apoptosis. Basic Res Cardiol 2009; 104:377-89.

15. Simpon PJ, Lucchesi BR. Free radicals and myocardial ischemia and reperfusion injury. J Lab Clin Med 1987; 110:13-30.

16. Adrie C, Laurent I, Monchi M, et al. Postresuscitation disease after cardiac arrest: a sepsis-like syndrome? Curr Opin Crit Care 2004; 10:208-12.

17. Kawakami R, Saito Y, Kishimoto I, et al. Overexpression of brain natriuretic peptide facilitates neutrophil infiltration and cardiac matrix metalloproteinase- 9 expression after acute myocardial infarction. Circulation 2004; 110:3306-12.

18. Weber M, Hamm C. Role of B-type natriuretic peptide (BNP) and NT-proBNP in clinical routine. Heart 2006; 92:843-9.

19. D'Souza SP, Yellon DM, Martin C, et al. B-type natriuretic peptide limits infarct size in rat isolated hearts via KATP channel opening. Am J Physiol Heart Circ Physiol 2003; 284:H1592-600.

20. Kasama S, Furuya M, Toyama T, Ichikawa S, Kurabayashi M. Effect of atrial natriuretic peptide on left ventricular remodelling in patients with acute myocardial infarction. Eur Heart J 2008; 29:1485-94.

21. Bainey KR, Armstrong PW. Clinical perspective on reperfusion injury acute myocardial infarction. Am Heart J 2014; 167:637-45.

22. Michaels AD, Klein A, Madden JA, Chatterjee K. Effects of intravenous nesiritide on human coronary vasomotor regulation and myocardial oxygen uptake. Circulation 2003; 107:2697-701. 